\title{
Aliénation et altérité : la construction identitaire dans Aliss de Patrick Senécal
}

\author{
Clotilde LANDAIS \\ Université de Pepperdine
}

En littérature contemporaine, comme en sociologie, en anthropologie, en psychanalyse ou en philosophie, la critique porte une attention particulière à des notions qui recoupent, toutes, la problématique de la construction identitaire: écritures migrantes, marginalité, différence, altérité ou encore aliénation. Cependant, la question de la construction de l'Autre en littérature repose encore fréquemment sur des stéréotypes sociaux, issus des mouvements de décolonisation et de migration. Pour cette raison, l'Autre est personnifié dans nombre de textes appartenant aux «littératures de l'altérité » sous les traits inquiétants d'un étranger, d'un vagabond ou 
encore d'un fou. Cependant, cette vision est réductrice, car elle ignore ce que Julia Kristeva, dans son essai intitulé Étrangers à nous-mêmes(1988), définit comme la nécessaire acceptation de l'étranger en nous-mêmes (p. 9). Dans son roman fantastique Aliss, l'auteur québécois Patrick Senécal s'attache à cette question à partir de l'altérité double -- c'est-à-dire à la fois objective et subjective -- d'une adolescente. En effet, comme le souligne Janet Paterson dans Figures de l'Autre dans le roman québécois, «[...] tout personnage fictif-- comme tout être humain -- peut se voir attribuer (ou s'attribuer soi-même) un statut d'altérité » (p.17). Si le genre fantastique n'appartient pas de façon consensuelle aux littératures de l'altérité, l’Autre en est paradoxalement le motif majeur, comme le relève la définition que donne Roger Caillois du fantastique :

La démarche essentielle du fantastique est l'apparition : ce qui ne peut pas arriver et qui se produit pourtant, en un point et à un instant précis, au cœur d'un univers parfaitement repéré et d'où on estimait le mystère à jamais banni. Tout semble comme aujourd'hui et comme hier: tranquille, banal, sans rien d'insolite, et voici que lentement s'insinue ou que soudain se déploie l'inadmissible. (p. 7)

Cet inadmissible est généralement le fait d'un Autre par excellence, le monstre -- diable, vampire, fantôme, loup-garou, pour ne reprendre que quelques-uns des thèmes du genre énumérés par Caillois (p.19-21). Dans Aliss, l'Autre n'est en aucun cas un monstre surnaturel, mais nous verrons que l'inscription du roman dans le genre fantastique renforce le propos de l'auteur sur l'altérité. Par ailleurs, bien que l'Autre de Senécal n'entre pas non plus dans les stéréotypes sociaux évoqués précédemment, la réflexion menée par l'auteur sur une altérité objective et subjective s'inscrit pourtant dans la 
continuité des littératures dites de l'altérité et de leurs théories, comme nous allons le voir dans cet article.

La définition de l'altérité sur laquelle nous baserons notre étude est celle qui part de la dualité inhérente au concept telle que relevée par François Hartog :

Dire l'autre, c'est le poser comme différent, c'est poser qu'il y a deux termes $a$ et $b$ et que a n'est pas b [...]. Mais la différence ne devient intéressante qu'à partir du moment où $a$ et $b$ entrent dans un même système. (p. 225)

Le système auquel fait référence Hartog correspond à ce qu'Éric Landowski dans Présences de l'autre appelle le « groupe de référence» (p.45-86). En effet, pour qu'un individu soit stigmatisé comme Autre, il faut une base de comparaison avec une norme. Cette norme est en général posée par un groupe majoritaire qui s'appuie sur ses propres codes sociaux et paramètres pour différencier, voire exclure l'Autre. Par exemple, dans le roman réaliste français du XIXe siècle, comme dans la société qu'il souhaitait refléter, le groupe de référence est constitué d'hommes blancs, occidentaux, hétérosexuels, de culture judéo-chrétienne, et toute personne présentant des traits constitutifs distincts de ce groupe - y compris les femmes - est alors identifiée comme Autre dans la narration (Schehr, 2003, p. x). Par ailleurs, dans la mesure où ce sont les valeurs décrites dans l'énonciation qui marquent la normalité $\mathrm{du}$ groupe de référence, celle-ci est nécessairement contextuelle, tout comme l'altérité qui en résulte. Ainsi, par le biais de stratégies narratives, des textes littéraires peuvent jouer sur l'existence de plusieurs groupes normatifs, et donc sur une altérité subjective parallèle à une altérité objective. C'est le cas du roman Aliss de Patrick Senécal, où l'héroïne passe d'une communauté à une autre en quête de son identité. 
Réécriture des contes de Lewis Carroll Alice's Adventures in Wonderland et Through the Looking-Glass and What Alice Found There, Aliss de Patrick Senécal offre un traitement littéraire original de l'altérité : en effet, nous verrons dans un premier temps que l'héroïne se construit un sentiment d'altérité à l'égard d'un groupe de référence avec lequel elle ne présente cependant aucun trait distinctif pertinent. Mais, bien qu'infondé, ce sentiment d'altérité amène l'héroïne à s'attacher à un second groupe de référence dont l'existence est possible grâce à l'inscription du récit dans le genre fantastique. Nous nous appliquerons donc, dans un deuxième temps, à montrer que ce second groupe est objectivement Autre, marginal, et que l'attitude de l'héroïne envers lui relève de l'aliénation. Enfin, dans un troisième temps, nous nous pencherons sur l'incapacité de l'héroïne à s'intégrer à ce second groupe de référence, ce qui la renvoie par là même à son statut d'Autre. Cependant, loin d'être un échec, nous verrons que ce passage par une altérité objective est nécessaire à la construction identitaire de l'héroïne.

\section{D’un groupe de référence à l'Autre}

Aliss présente une adolescente québécoise vivant dans la banlieue bourgeoise de Montréal, d'où elle est originaire. S'appuyant sur les mécanismes du récit réaliste, la narration désigne l'entourage direct de l'héroïne comme groupe de référence du roman. Les parents, amis et professeurs de l'héroïne appartiennent en effet à la population majoritaire du Québec, à savoir des individus blancs de langue française, hétérosexuels et de culture judéo-chrétienne. Les premières 
pages du roman, sous forme de témoignages des représentants de ce groupe, indiquent que l'héroïne y est ancrée : Alice/Aliss, narratrice autodiégétique du récit, est une Québécoise de souche qui évolue dans un milieu aisé et suit des cours au Cégep. Bien qu'un peu rebelle, elle est bonne élève, comme le souligne son petit ami, issu du même groupe :

Le genre de fille qui n'a pas peur de s'affirmer. Rebelle, mais pas conne. On s'est parlé durant le party de mi-session pis... on sort ensemble depuis ce temps-là. Ça fait presque deux mois. C'est une fille studieuse, qui vient d'une famille riche, mais elle est pas straight pour autant... Pis cultivée! Elle lit beaucoup, écoute toutes sortes de musiques, toutes sortes de films... même des films européens! (p. 2-3)

Ainsi, contrairement aux récits traditionnels de l'altérité mettant en scène un vagabond ou un étranger, l'héroïne de Senécal ne présente aucun trait pertinent la distinguant de la norme posée par le groupe de référence. Bien qu'elle soit qualifiée à plusieurs reprises de «rebelle» ou de «contestataire» (p. 4) par ses proches, ces traits sont neutralisés dans le discours par une appartenance non ambiguë au milieu intellectuel du groupe normatif. Ceci est notamment souligné par le fait qu'elle est «studieuse», «cultivée » et ouverte à différentes cultures. La présentation qui est faite de l'héroïne par les représentants du groupe de référence du roman ne la situe donc absolument pas comme Autre.

Pourtant, l'adolescente traverse une crise identitaire telle que cela engendre en elle un sentiment d'altérité suffisant pour qu'elle quitte le domicile parental :

Je leur ai dit que je me posais plein de questions, depuis quelque temps, que je réfléchissais beaucoup. [...] Je leur ai dit que je les aimais, que j'aimais mes amis, que j'haïssais pas non plus la vie que je menais, mais [...] qu'il fallait que je connaisse 
autre chose. Parce qu'il y a d'autres choses, je le sais. D'autres possibilités de vie, d'autres moyens d'envisager l'existence. Je veux expérimenter l'ailleurs, aller au bout de moi-même. Qu'est-ce qui existe, à l'extérieur de la famille, des amis et de l'école? Et surtout, qu'est-ce qui existe en dehors des conventions? En dehors des règles? En dehors du conformisme? (p. 8)

Ce passage souligne de façon significative les attributs du groupe de référence tels que définis par Landowski : la famille, les amis et l'institution scolaire sont mis en parallèle avec les conventions, les règles établies par le cercle en question. Si l'héroïne reconnaît se sentir bien au sein de ce groupe, elle est également curieuse de tester ses limites et celles de la société; elle souhaite sortir du « conformisme » imposé par son milieu social, vivre « autre chose » :

L'altérité peut [...] impliquer une construction de la part d'un sujet à l'égard d'un groupe de référence. Dans ce cas, c'est l'individu qui tient à se distinguer du groupe de référence auquel il ne veut pas appartenir, c'est lui ou elle qui marque sa différence de traits pertinents. (Paterson, 2004, p. 26-27)

Ainsi, sans raison apparente, l'héroïne se construit subjectivement comme Autre au sein de son groupe de référence. Elle cherche alors un second groupe normatif auquel s'identifier, et cette quête d'un nouveau groupe de référence se fait par l'expérimentation de ce qu'Aliss appelle "l'ailleurs » (p. 8). Or, comme le relève l'ensemble des théories de l'altérité, le changement d'espace, le franchissement d'une frontière est capital pour la construction de l'altérité d'un personnage. Cependant, l'héroïne du roman éponyme ne cherche nullement à quitter son pays, ni même sa ville; comme les figures traditionnelles de l'altérité, elle souhaite s'affranchir de sa famille (Kristeva, 1988, p.13), mais sans l'exil radical qui 
accompagne généralement cette libération; elle ne souhaite que traverser le fleuve. Toutefois, par le jeu du fantastique, ce franchissement de frontière prend une dimension similaire à celle d'un exil: tout comme dans le premier conte de Carroll, Alice's Adventures in Wonderland, la migrance ontologique de l'héroïne est signifiée dans la narration par le franchissement d'un tunnel - celui du métro, ce qui établit immédiatement un parallèle avec l'univers de Carroll : « Je tente quand même de deviner le nom de la station, malgré le peu de lettres encore visibles: W D R D.» (Senécal, 2000, p. 26) Comme l'héroïne de Carroll, celle de Senécal est bel et bien coupée de son monde. En effet, le fantastique transporte l'adolescente dans un espace-temps parallèle à celui du groupe de référence du roman. Elle y est retenue contre sa volonté, car seul le métro peut lui permettre de retourner dans son monde (p. 165), symbolisé par le pont Jacques-Cartier qu'elle voit du Wonderland, mais elle ne pourra le reprendre qu'une fois sa quête identitaire achevée (p.514). Cette proximité apparente avec le groupe de référence du roman ne limite donc en rien l'expérience de l'altérité que vit l'héroïne, car, grâce au fantastique, le Wonderland constitue une "spatialité distincte de celle du groupe de référence » (Paterson, 2004, p. 29). Le fait que l'héroïne se trouve dans une situation réelle d'exil, comme dans les littératures traditionnelles de l'altérité, l'empêche de renoncer à sa quête identitaire lorsque les premières difficultés surgissent (p. 169) et la condamne à vivre l'expérience jusqu'au bout, à « aller au bout [d'elle]-même » (p. 8).

Ainsi, l'héroïne du roman éponyme est menée par ses questions existentielles à un univers clos, un monde parallèle dans lequel les personnages imaginés par Lewis Carroll se sont transformés en criminels en fuite et déviants sexuels (Landais, 
2009) : Charles - l'auteur -, est un mathématicien pédophile (p. 277); la reine de Cœur, devenue la reine Rouge, est une meurtrière récidiviste (p. 424-427); la Duchesse, sous le nom d'Andromaque, est la prostituée la plus en vue du quartier (p. 188); quant au Chapelier et au Lièvre de Mars, les personnages les plus excentriques de Carroll, ils se retrouvent sous les traits de Chair et Bone, des tortionnaires au service de la souveraine (p. 305). Dans la mesure où la formation discursive de l'Autre repose sur des stéréotypes sociaux (Paterson, 2004, p. 20), de telles caractéristiques, même dans un texte fantastique, construisent narrativement les habitants $\mathrm{du}$ Wonderland comme des figures de l'Autre : l'onomastique tout d'abord, qui renvoie soit à la violence corporelle, comme le duo Chair et Bone, soit à la laideur physique, comme Verrue : «Sa face a été labourée par tous les tracteurs du monde. Il y a tellement de rides que j'ai peine à distinguer sa bouche fermée. Son nez est long et pendant, une masse de chair vide » (p. 46); les valeurs négatives qui leur sont attribuées par le texte ensuite, comme leur comportement dénué de tout tabou ou leur absence de morale : «Y a pas de place pour [une] morale [ici]! Y a pas de place pour la philosophie, pour l'homme meilleur, pour comprendre qui on est! » (p. 505); les traits langagiers de certains habitants du quartier enfin, comme les nombreux « sacres » et expressions québécoises populaires de la reine ou les alexandrins d'Andromaque, qui placent ces personnages en dehors du groupe de référence du roman, au français plus conventionnel et soutenu, et font vivre à Aliss l'expérience langagière de l'altérité :

- Bon, c'est pas impossible que j'te prenne sous mon aile, Comme ça, tout habillée, tu m'as l'air assez belle. [...] 
J'approuve en silence, fascinée par sa façon de parler, partagée entre le fou rire et l'admiration. [...]

- Quel âge que t'as, au juste? Vingt et un? Vingt-deux ans?

T’as l'air jeune : un bon point. Ça, ça plaît aux clients.

C'est incroyable, elle parle vraiment comme ça naturellement! (p. 186)

Cependant, si les personnages pervertis de Carroll représentent l'Autre aux yeux du groupe de référence du roman, au sein du Wonderland, ces marginaux sont le groupe de référence.

\section{L'aliénation ou le refus de reconnaître une altérité objective}

Isolés dans leur univers clos grâce à l'inscription du roman dans le fantastique, les habitants du Wonderland ont fondé une société qui leur est propre et ont élu une souveraine qui prend à cœur ses devoirs envers son peuple, comme l'illustre le passage suivant :

[L]a Reine pointe un doigt fatal vers Mario et hurle :

- Pis pour avoir voulu défier ce pouvoir, je te condamne, Mario, à mourir par les mains du peuple! Ce peuple qui est pas victime de mon pouvoir, mais qui en profite! [...] Meurs maintenant!

Nouveau délire de joie dans mon dos. [...] Même si la planche sur laquelle est attaché Mario me fait dos, je vois les dizaines de fléchettes voler vers elle, lancées avec rage et euphorie [...] et les dards volent toujours, et les cris, et les applaudissements [...]. (p. 506)

La reine Rouge règne donc sur le quartier; elle en établit les codes sociaux et les paramètres, qui sont acceptés par la population, comme en attestent le lancement euphorique de fléchettes et les applaudissements. Or, comme nous l'avons 
signalé précédemment, toute altérité est variable et tributaire d'un processus de construction idéologique (Paterson, 2004, p. 27). Ainsi, bien que les habitants du Wonderland représentent l'Autre aux yeux du groupe de référence du roman, ils forment parallèlement un ensemble qui est construit narrativement comme groupe de référence au sein de ce quartier isolé par le fantastique.

Aussi, si l'on confronte les traits constitutifs des deux groupes de référence présents dans le roman de Senécal, il apparaît que l'héroïne est bien construite comme Autre par rapport aux habitants du Wonderland. Cependant, en quête d'expérimentation, à la recherche de ce qui existe " en dehors des conventions » (Senécal, 2000, p. 8), Aliss se force à oublier ses valeurs et son éducation, et tente d'adhérer à la mentalité du lieu :

Mais c'est interdit, des shows comme ça, non? La police doit pas être au courant certain!

En tout cas, interdit ou non, faut avouer que c'est quand même pas désagréable...

Je ricane. Je commence à pogner le beat, on dirait. C'est bon, ça. Très bon! (p. 93)

Un tel raisonnement chez l'héroïne montre qu'elle refuse de se sentir Autre dans ce quartier qui correspond à ce qu'elle croit chercher. Cependant, en niant que le comportement des habitants du quartier la choque, l'héroïne entre dans un processus d'aliénation.

L'aliénation se définit généralement comme le fait de devenir étranger à soi-même au profit de forces supérieures. L'aliénation de l'héroïne à ceux qu'elle considère comme constitutifs de son groupe de référence se lit alors dans tous ses efforts d'intégration : le changement d'orthographe de son nom 
tout d'abord (p. 35); ensuite, l'adoption d'un style vestimentaire plus conforme au quartier (p.76); et enfin, l'abandon, une à une, de ses valeurs, au fil de sa «libération" sexuelle. Ce processus rejoint ce que Julia Kristeva considère comme une conséquence de l'exil :

S'arracher à sa famille, à sa langue, à son pays, pour venir se poser ailleurs, est une audace qu'accompagne une frénésie sexuelle: plus d'interdit, tout est possible. Peu importe si le passage de la frontière est suivi d'une débauche ou, au contraire, d'un repli peureux. Toujours l'exil implique une explosion de l'ancien corps, un poudroiement. (p. 47)

Cette dialectique de la frontière, que nous avons déjà mentionnée à l'occasion du déplacement spatial inhérent à la notion d'altérité, est clairement liée dans le roman à celle de la libération sexuelle recherchée par Aliss et ce, à travers le personnage de Verrue :

Pour aller au bout de soi-même, il faut franchir plusieurs étapes... Chaque étape est une frontière qui nous amène un peu plus loin. Il s'agit de découvrir quelle frontière nous arrêtera... C'est cette frontière qui nous révèlera à nous-mêmes. (p. 72)

Ce passage souligne que chaque étape de la libération sexuelle de l'héroïne, dont découle cette « explosion de l'ancien corps » mentionnée par Kristeva, est perçue par Aliss comme le franchissement d'une frontière vers l'intégration. La première frontière franchie est celle d'un rapport sans protection (p. 105108). La deuxième est celle des drogues dures. Aliss consommait des drogues douces avant son arrivée au Wonderland (p.3), mais elle est assez sceptique quant aux pilules. Cependant, après son expérience sexuelle et forte de sa lecture de Ainsi parlait Zarathoustra, elle fait taire ses inquiétudes : 
Je [...] tombe sur les deux petits flacons de pilules, les Micros et les Macros.

Je les regarde longuement.

De la drogue chimique. Que je connais pas.

J'ouvre le flacon de Micros. Prends une pilule. L'étudie avec attention, comme si je m'attendais à trouver la liste des ingrédients inscrite dessus.

J'ai peur comme c'est pas possible. Et cette peur m'excite au plus haut point.

La vertu dans l'action, hein, mon Nietzsche?

J'avale la Micro. Gloup. (p. 110)

Les phrases courtes de ce passage reflètent l'hésitation de l'héroïne, une hésitation nourrie par tout ce qu'elle a pu lire ou entendre sur les drogues dures. Mais une fois encore, elle a décidé de s'intégrer et de faire "comme tout le monde », quel que soit le prix de cette décision. Les railleries de Verrue et les citations de Nietzsche ont raison de son inquiétude. Le franchissement de cette deuxième frontière lui permet d'aller plus avant dans sa libération sexuelle, puisqu'elle commence à travailler comme strip-teaseuse au club d'Andromaque. La dernière frontière franchie par Aliss, devenue dépendante de la drogue, est la prostitution. La libération sexuelle de l'héroïne est donc achevée, tout comme l'explosion de son ancien corps qui résulte de cette frénésie sexuelle, mais cela n'a pu se faire que sous l'emprise de la drogue. Cette nécessité pour Aliss de se droguer afin de se libérer de ses inhibitions révèle un écart entre son moi réel et celui qu'elle souhaite projeter. Cet écart est par ailleurs signifié dans la narration par la " petite voix» qui se fait entendre avant chaque franchissement de frontière :

- Agenouille-toi! qu'il souffle.

[...] Non! fait une petite voix en moi. Non, pas ça, quand même!

Â ma grande surprise, je m'agenouille. [...] Je reste à genoux. Je fais rien, je dis rien, je bouge pas, passive et fascinée. Fascinée par ça, fascinée par moi-même en personne. (p. 105-108) 
Cette voix est celle de la vraie Alice, et son intervention souligne la répression de son identité réelle que s'impose l'héroïne dans l'espoir d'être assimilée par ce groupe de référence. Par le décalage qu'elle induit entre les actes de l'héroïne et son vrai moi, la «petite voix» signale donc l'aliénation de l'héroïne.

Ce décalage est également signifié dans la fascination qu'Aliss éprouve pour elle-même, pour cette Autre qui se prête à des actes dont elle ne se serait jamais cru capable. En effet, l'Autre est source de fascination car, comme le souligne Janet Paterson :

[C]'est au-delà des systèmes visibles d'opposition, qui régissent la construction de l'Autre, que réside fréquemment la puissance signifiante de l'altérité. Basé sur un rapport oppositionnel, le discours a beau multiplier les stratégies de mise en différence entre le soi et l'Autre, il a beau insister sur l'écart qui les sépare, quelque part dans le texte une vérité se fait entendre : ce qui s'oppose se relie par la force même de la logique binaire. [...] L'Autre en soi, le soi en l'Autre, voilà ce qui régit en réalité la relation identité/altérité dans ses aspects les plus menaçants [...] ou idéalisants [...]. (p. 38)

Par le biais du fantastique, l'incarnation de cet Autre en soi est possible. En effet, bien qu'elle ne l'identifie pas alors comme tel, l'héroïne en a conscience dès son arrivée au Wonderland :

Ah! Tiens, il y a quelqu'un de l'autre côté, presque au bout du quai, là-bas. Une fille. Habillée d'un bustier et d'une jupe courte. Elle a l'air dans un sale état. Ses vêtements sont en lambeaux et ses cheveux anarchiques. Des cheveux bruns et longs, comme les miens. Je me demande même si je ne perçois pas des traces de sang sur son visage et ses jambes. Elle est assise, ou plutôt écrasée sur un banc. Elle semble à peu près de ma grandeur. Le même âge que moi, ou à peu près... 
[...] Je ne la distingue pas parfaitement, mais on dirait vraiment que...

[...] Il faut que j'en aie le cœur net! [...] Mais voilà que le métro arrive de l'autre côté et me cache la fille. Je me mets à courir. Il faut au moins que je la voie à travers la vitre du wagon! [...]

Un peu essoufflée, je finis par hausser les épaules. Bon. Tant pis. J'imagine que si je l'avais vue de près, la ressemblance aurait été moins frappante... (p. 22-23)

Grâce au fantastique et à une distorsion temporelle, l'héroïne voit donc son double et son futur. Comme en atteste ce passage, son corps porte les traces de l'exil, de l'« explosion de [son] ancien corps ». Ainsi, c'est bien son Autre en soi qu'Aliss découvre à travers son aliénation au groupe de référence du Wonderland, et le fantastique permet une représentation physique de ce dédoublement qui «signale la division primordiale : c'est-à-dire la coexistence du conscient et de l'inconscient. » (Delvaux, 1995, p. 490) C'est cet Autre en soi qui, par le biais des drogues dures, fait taire le conscient de l'héroïne :

- Écoute, Verrue, je voulais te dire : j'ai été engagée comme danseuse chez Andromaque. Je commence à soir. [...] Pourtant, l'audition a été un... C'était un désastre, vraiment. J'étais humiliée, gênée, je me trouvais pourrie, pis... [...] mais je sais que je peux le faire. Avec de la Macro, je le peux... Ça me libère, tu comprends?

— Ça te libère de quoi? [...]

— Ça me libère... de moi... de mon ancien moi [...]. (p. 206)

Ce passage révèle que, par l'action de l'Autre en soi, l'aliénation de l'héroïne au groupe de référence est désormais totale. Loin de voir comme un signe d'aliénation la nécessité de se droguer pour danser nue puis se prostituer, Aliss perçoit sa capacité à «faire comme tout le monde » comme une libération. La «petite voix» de sa conscience ne peut désormais plus 
s'exprimer pour souligner le décalage entre les valeurs de l'adolescente et celles des habitants du quartier. Cependant, l'action de son Autre en soi empêche également l'héroïne de se rendre compte que, malgré tous ses efforts, elle n'est pas pour autant acceptée au Wonderland. Ainsi, alors qu'elle franchit une à une lesdites frontières, croyant s'intégrer, les autres membres de la communauté voient son statut d'Autre s'affirmer.

\section{Échec de l'assimilation et victoire de l'identité}

C'est le rôle de la reine d'apprendre à l'héroïne que ses tentatives pour être assimilée ont échoué. En effet, c'est elle qui organise les épreuves auxquelles se soumet (à son insu) l'héroïne, dans le but de la guider dans sa quête identitaire (Landais, 2008) :

- Je voulais que tu voies tout, que tu vives toutes les étapes, jusqu'au viol final! Parce qu'évidemment je le savais que t'oserais pas tuer Mickey et Minnie! [...] Sais-tu pourquoi? [...] Parce que je savais que t'étais pas faite pour vivre ici! Que t'étais pas des nôtres! (p. 501)

Ainsi, bien que l'héroïne, en aliénant son identité, fasse son possible pour être acceptée par ce groupe comme l'une des leurs, les habitants du Wonderland l'objectivent comme Autre. Cette altérité objective de l'héroïne est révélée lors de l'épreuve finale, qui consiste en une exécution et qui provoque un sursaut des valeurs auxquelles croit l'héroïne. L'interdiction de tuer est si fortement ancrée en elle que même la combinaison « droguevengeance-aliénation" ne peut la lui faire oublier. Or, c'est précisément parce que l'héroïne aliène son identité à son Autre 
en soi, parce qu'elle veut être ce qu'elle n'est pas, qu'elle est exclue du quartier :

- Toi, ce que t'as fait depuis ton arrivée ici, l'as-tu fait vraiment par conviction? Te sentais-tu toujours authentique? Non! Tu l'as fait parce que tu croyais qu'il fallait faire ça pour être libre, tu voulais t'en convaincre! [...] Ton Nietzsche a pourtant dit une phrase importante: "Deviens ce que tu es!" En venant ici, t'es pas devenue ce que tu es fondamentalement, Aliss : t'as tenté de devenir ce que tu n'es pas!(p. 503)

Ce passage renvoie à ce que Julia Kristeva appelle «le paradoxe du comédien »: «[...] multipliant les masques et les "faux selfs", il n'est jamais tout à fait vrai ni tout à fait faux » (p.18). Aliss tente de dissimuler son vrai moi, mais les habitants du Wonderland perçoivent la superficialité de son comportement et la démasquent. Cependant, ce passage souligne également le fait que le refus du groupe d'assimiler l'héroïne ne se place pas dans la logique de ce que Landowski appelle un "discours d'exclusion», dans la mesure où il ne procède pas « d'un geste explicitement passionnel tendant à la négation de l'Autre en tant que tel » (p. 22). Contrairement à la réaction de nombreux groupes de référence face à l'altérité, la population du Wonderland ne craint pas Aliss. Elle est Autre, au sens où elle représente la société que ces marginaux ont fuie, mais elle ne les menace pas car elle ne renvoie à aucune «face cachée de [leur] identité » (Kristeva, 1988, p. 9). Pour cette raison, leur attitude est plutôt celle d'adjuvants. Certes, leur aide peut paraître paradoxale, puisqu'ils la poussent à se droguer et à se prostituer; malgré tout, il s'agit pour les habitants du quartier de guider l'héroïne dans sa quête identitaire, de l'aider à se construire en opposition à cet Autre qu'ils savent constituer par rapport au groupe de référence du roman. En effet, comme le souligne Éric Landowski, la mise en 
avant de l'écart qui existe entre l'Autre et le groupe normatif provoque généralement une prise de conscience identitaire de la part de l'Autre :

[U]n sujet ne peut au fond se saisir lui-même en tant que " Je", ou que "Nous», que négativement, par opposition à un "Autre» qu'il lui faut alors construire comme figure antithétique afin de pouvoir se poser lui-même, comme son contraire. [...] Pour fonder sa propre certitude d'être Soi, la seule chose qui importe, la seule "vérité » dont il lui faille s'assurer, c'est que l'Autre est «autre», et qu'il l'est catégoriquement. (p. 40)

La connaissance de soi passe donc par celle de l'Autre. C'est-à-dire que l'Autre construit son identité en négatif de celle du groupe de référence. C'est en ce sens que la reine présente les épreuves successives à l'héroöne, afin de mettre en évidence tout ce qui oppose l'adolescente aux habitants du quartier. L'héroïne doit ainsi comprendre qu'ils sont «Autres» par rapport à elle et qu'elle appartient au groupe de référence du roman. C'est la raison pour laquelle la reine souligne que les valeurs «de l'amour, du respect, de l'entraide» (p. 500) auxquelles l'héroïne croit, mais qu'elle s'est efforcée de faire taire lors de son aliénation, n'ont pas cours dans le quartier. Contrairement à elle, les habitants du quartier et leur reine savent qui ils sont et l'acceptent :

Un tonnerre d'applaudissement explose derrière moi et tous se mettent à scander Vive la Reine Rouge! Vive la Reine Rouge! Je les regarde, abasourdie. Dans leurs cris, sur leurs visages, il y a cette certitude absolue, cette assurance indicible, cette parfaite conscience de ce qu'ils sont! Et, surtout, de ce qu'ils ne sont pas! (p. 506)

L'énonciation autodiégétique reflète ici la prise de conscience de son altérité par l'héroïne par rapport à ce groupe, 
ce qui achève par là même sa construction identitaire. Comme le lui dit la reine, elle est " parfaitement... normale » (p. 508) et a sa place dans le groupe de référence du roman :

- [L]es gens comme toi sont prévus dans la société de là-bas. Ils sont, si on veut, le Parti de l'opposition qui contribue au fonctionnement du système, parce que, tout en brassant de la marde, ils s'intègrent. Les gens comme toi veulent changer la formule. Pourtant, ils font partie de l'équation. (p. 507)

Aliss peut donc retourner au sein du groupe de référence du roman, représenté par ses parents, amis et professeurs. Son sentiment d'altérité, qui s'était manifesté en l'absence de tout trait différentiel et qui l'avait conduite à aliéner son identité en faveur d'une altérité objective, disparaît, comme le souligne Julia Kristeva : «[...] l'étranger commence lorsque surgit la conscience de ma différence et s'achève lorsque nous nous reconnaissons tous étrangers, rebelles aux liens et aux communautés» (p. 9). La reconnaissance de son altérité objective au sein du groupe de référence du Wonderland permet en effet à l'héroïne de s'accepter pleinement-y compris la part d'Autre en soi - et de rejoindre le groupe de référence du roman. Elle sait désormais qu'elle n'y est pas Autre, mais qu'elle est simplement différente - sans contenu spécifique, contrairement à l'altérité :

[Ê]tre soi-même, ce n'est pas seulement être, ou s'affirmer, « autre que l'Autre ». C'est en même temps un peu plus que cela. Au minimum, c'est aussi, tout bêtement, « exister » (plutôt que ne pas être) [...]. C'est « vivre » en donnant, s'il se peut, un sens à ce que l'on fait de sa propre vie ou, à défaut, en tentant de comprendre ce que "la vie» elle-même fait de nous. (Landowski, 1997, p. 41)

«Vivre » et trouver un sens à sa vie correspondent à ce que souhaitait découvrir l'héroïne en quittant le groupe de 
référence du roman pour « expérimenter l'ailleurs, aller au bout d'[elle]-même » (Senécal, 2000, p. 8). Mais grâce à son expérience d'une altérité objective, elle a également appris à être elle-même et à s'accepter.

\section{Conclusion}

Aliss décrit le processus par lequel un personnage hors de tout stéréotype social se construit une altérité subjective, tout en niant une altérité objective à laquelle il s'aliène. Le roman présente donc un processus de construction identitaire à la fois romanesque et philosophique, au sens donné par Paul Ricœur (1990), car l'héroïne forme sa propre identité par rapport à autrui. Par le jeu entre deux groupes de référence, Patrick Senécal montre que si l'altérité relève bien d'un processus de construction idéologique (Paterson, 2004, p. 27), elle s'avère toutefois nécessaire à la constitution d'une identité. De plus, même subjective, l'altérité peut conduire un sujet à une aliénation aussi totale qu'une altérité objective. Senécal prolonge par là même les définitions classiques de l'altérité en littérature, tout en confirmant l'aspect postmoderne de l'interrogation en la matière relevée par Jean-François Lyotard (1979) en insérant cette réflexion, non dans un métarécit, mais dans une littérature dite de marge, le fantastique.

\section{Bibliographie}

CAILloIS, Roger (1966), Anthologie du fantastique 1, Paris, Gallimard. 
CARRoll, Lewis (1960 [1865 et 1871]), The Annotated Alice:Alice's Adventures in Wonderland and Through the Looking-Glass and What Alice Found There, MartinGARDNER (éd.), Harmondsworth, Penguin.

DelvauX, Martine (1995), «Le Moi et l'A/autre: subjectivité divisée et unité culturelle ", Revue canadienne de littérature comparée, vol. 22, nos 3-4, p. 487-500.

HARTOG, François (1980), Le Miroir d'Hérodote. Essai sur la représentation de l'autre, Paris, Gallimard.

KrISTEVA, Julia (1988), Étrangers à nous-mêmes, Paris, Gallimard, coll. « Folio essais ».

LANDAIS, Clotilde (2008), «Aliss de Patrick Senécal : le corps violenté comme représentation métatextuelle», dans Julie Delorme et Claudia Labrosse (dir.), «Écriture du corps, corps de l'écriture », @nalyses, p. 20-34.

LANDAIS, Clotilde (2009), " L'hypertextualité comme révélateur d'identité dans Alissde Patrick Senécal », Clair-Obscur, vol. 5, p. 50-61.

LANDOWSKI, Éric (1997), Présences de l'Autre. Essais de sociosémiotique II, Paris, Presses universitaires de France.

LYOTARD, Jean-François (1979), La Condition postmoderne, Paris, Minuit, coll. « Critique ».

PATERSON, Janet (2004), Figures de l'Autre dans le roman québécois, Québec, Nota Bene, coll. «Littérature(s) ».

RICOEUR, Paul (1997 [1990]), Soi-même comme un autre, Paris, Seuil, coll. «Points Essais ».

SCHEHR, Lawrence R. (2003), Figures of Alterity: French Realism and Its Others, Stanford, Stanford University Press.

SENÉCAL, Patrick (2000), Aliss, Québec, Alire, coll. « Fantastique ». 


\title{
Résumé
}

Le roman Aliss de l'auteur québécois Patrick Senécal décrit le processus par lequel un personnage hors de tout stéréotype social se construit une altérité subjective, tout en niant une altérité objective à laquelle il s'aliène. En inscrivant son texte dans le genre fantastique et par un jeu entre deux groupes de référence, Senécal montre que, si l'altérité relève bien d'une construction idéologique, elle s'avère nécessaire à la constitution d'une identité. De plus, même subjective, l'altérité peut conduire le sujet à une aliénation aussi totale que celle suscitée par une altérité objective.

\begin{abstract}
Quebec writer Patrick Senécal's novel,Aliss,depicts the subjective alterity towards a normative group constructed by a character standing apart from all social stereotypes, while denying an objective alterity towards a second group. Through this play between two normative groups and resorting to fantastic fiction techniques, Senécal signifies that if alterity is indeed an ideological figure, it is necessary to create one's identity. Moreover, even a subjectively constructed feeling of alterity can lead a subject to an alienationas totalas the one caused by an objective alterity.
\end{abstract}

